\title{
Tumor suppressor in lung cancer-1 (TSLC1) mediated by dual-regulated oncolytic adenovirus exerts specific antitumor actions in a mouse model
}

\author{
Wen LEI ${ }^{1, \#}$, Hong-bin LIU ${ }^{1, \#}$, Shi-bing WANG ${ }^{1}$, Xiu-mei ZHOU ${ }^{1}$, Shui-di ZHENG ${ }^{1}$, Ke-ni GUO ${ }^{1}$, Bu-yun MA ${ }^{1}$, Yu-long XIA ${ }^{1}$, \\ Wen-song TAN ${ }^{2}$, Xin-yuan LIU ${ }^{1,3}$, Yi-gang WANG ${ }^{1,2, *}$ \\ ${ }^{1}$ Xinyuan Institute of Medicine and Biotechnology, College of Biological Sciences, Zhejiang Sci-Tech University, Hangzhou 310018, \\ China; ${ }^{2}$ State Key Laboratory of Bioreactor Engineering, East China University of Science and Technology, Shanghai 200237, China; \\ ${ }^{3}$ State Key Laboratory of Cell Biology, Institute of Biochemistry and Cell Biology, Shanghai Institutes for Biological Sciences, Chinese \\ Academy of Sciences, Shanghai 200031, China
}

Aim: The tumor suppressor in lung cancer-1 (TSLC1) is a candidate tumor suppressor of lung cancer, and frequently inactivated in primary non-small cell lung cancer (NSCLC). In this study, we investigated the effects of TSLC1 mediated by a dual-regulated oncolytic adenovirus on lung cancer, and the mechanisms underlying the antitumor actions.

Methods: The recombinant virus Ad.sp-E1A $\mathrm{A}_{(\Delta 24)}-\mathrm{TSLC1}$ was constructed by inserting the TSLC1 gene into the dual-regulated Ad.sp$\mathrm{E} 1 \mathrm{~A}_{(\Delta 24)}$ vector, which contained the survivin promoter and a $24 \mathrm{bp}$ deletion within E1A. The antitumor effects of Ad-sp-E1A $\mathrm{A}_{(\Delta 24)} \mathrm{TSLC1}^{-\mathrm{T}}$ were evaluated in $\mathrm{NCl}-\mathrm{H} 460, \mathrm{~A} 549$, and $\mathrm{H} 1299$ lung cancer cell lines and the normal fibroblast cell line MRC-5, as well as in A549 xenograft model in nude mice. Cell viability was assessed using MTT assay. The expression of TSLC1 and activation of the caspase signaling pathway were detected by Western blot analyses. The tumor tissues from the xenograft models were examined using H\&E staining, IHC, TUNEL, and TEM analyses.

Results: Infection of A549 lung cancer cells with Ad.sp-E1A ${ }_{(\Delta 24)}-T_{S L C 1}$ induced high level expression of TSLC1. Furthermore, the Ad.sp-

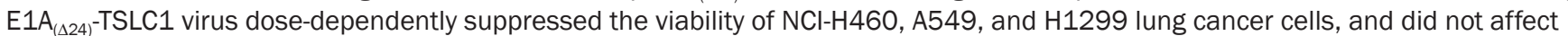

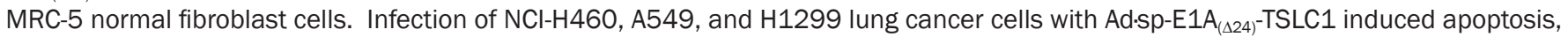
and increased activation of caspase-8, caspase-3 and PARP. In A549 xenograft model in nude mice, intratumoral injection of Ad.sp-

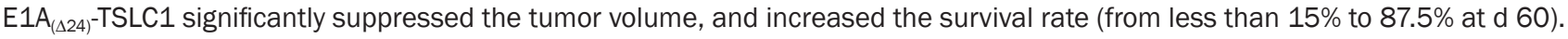
Histological studies showed that injection of Ad-sp-E1A ${ }_{(\Delta 24)}-\mathrm{TSLC1}$ caused tumor cell apoptosis and virus particle propagation in tumor tissues.

Conclusion: The oncolytic adenovirus Ad.sp-E1A $\mathrm{A}_{(\Delta 24)}-\mathrm{TSLC1}$ exhibits specific antitumor effects, and is a promising agent for the treatment of lung cancer.

Keywords: lung cancer; tumor suppressor in lung cancer-1; oncolytic adenovirus; survivin; apoptosis; caspase signaling pathway; tumor xenograft model

Acta Pharmacologica Sinica (2013) 34: 531-540; doi: 10.1038/aps.2012.196; published online 18 Mar 2013

\section{Introduction}

Although major progress has been made in the diagnosis and treatment of lung cancer, the five-year survival rate is only $14 \%$, which is largely because of the failure of cancer debulking surgery and systemic chemotherapy ${ }^{[1]}$. According to the Cancer Statistics Review, which was conducted between 1975

\footnotetext{
\#These authors contributed equally to this paper.

* To whom correspondence should be addressed.

E-mail wangyigang43@163.com

Received 2012-07-28 Accepted 2012-12-20
}

and 2009 by the SEER (Surveillance, Epidemiology and End Results) group at the National Cancer Institute in the USA, 219440 patients were estimated to have primary lung and bronchial tumors in 2009, and 158350 were estimated to have died as a result. Lung and bronchial tumors ranked the highest of all cancers in both the number of cases and the number of deaths. Currently, lung cancer is the most common primary malignant tumor, with over 1 million deaths worldwide occurring annually; this may in part be due to the increase in the population of smokers and environmental pollution. Thus, novel treatment methods for lung cancer are urgently needed, 
and these treatments must be highly effective and safe.

The Cancer Targeting Gene-Viro-Therapy (CTGVT) strategy developed by our group is one of the most promising approaches for cancer therapy ${ }^{[2]}$. The strategy combines the advantages of both gene therapy and virotherapy by using an oncolytic adenoviral vector (OA) harboring an anti-cancer gene. Currently, two main strategies are applied to construct the viral vector with oncolytic ability, which can specifically infect tumor cells but rarely infects normal cells ${ }^{[3]}$. The first strategy is to delete the adenoviral genes that are necessary for viral replication in normal cells but not in tumor cells. For example, the ONYX-015 and ZD55 oncolytic viruses were constructed by deleting the $E 1 B$ gene, which functions in late viral RNA export ${ }^{[4]}$. The second strategy involves transcription targeting through the use of tumor- or tissue-selective promoters, which can control the expression of early viral genes such as E1A and/or E1B that are essential for replication. Our previous studies have shown that CTGVT exhibits greater antitumor effects than gene therapy or virotherapy alone ${ }^{[3,5,6]}$.

The tumor suppressor in lung cancer-1 (TSLC1) was originally identified as a putative tumor suppressor for non-smallcell lung cancer (NSCLC) and was the first named tumor suppressor in lung cancer ${ }^{[7]}$. It is expressed in a variety of tissues and organs in the human body, particularly in the normal lung, brain, liver and skin ${ }^{[8]}$. The downregulation of the TSLC1 gene was frequently detected in various human cancers including gastric cancer ${ }^{[9-11]}$, hepatocellular carcinomas ${ }^{[12]}$, cervical cancer ${ }^{[13]}$, nasopharyngeal cancer ${ }^{[14]}$, breast cancer ${ }^{[15]}$, prostate cancer ${ }^{[16]}$, and pancreatic cancer ${ }^{[17]}$. TSLC1 is a transmembrane adhesion molecule that belongs to the immunoglobulin superfamily ${ }^{[18]}$, and it consists of an extracellular domain (EC), a transmembrane domain (TM) and a cytoplasmic domain (CP). The EC of TSLC1 mediates the formation of TSLC1 homodimers or heterodimers with other cell adhesion molecules, such as Necl-1, CRTAM, and Nectin-3, to regulate cell-cell adhesion. The CP interacts with DAL-1, another tumor suppressor gene, and membrane-associated guanylate kinase (MAGuK) homologs such as MPP3. The CP is able to regulate the activation of small Rho GTPases, thus acting as a vital connection between extracellular adhesion and intracellular signaling cascades. Furthermore, the possible molecular mechanisms of TSLC1 include the suppression of tumor formation, modulation of the cell cycle, pro-apoptotic activity and regulation of the epithelial-mesenchymal transition $(\mathrm{EMT})^{[19]}$.

Human survivin, the smallest member of the inhibitor of apoptosis protein (IAP) family, plays a key role in both the regulation of cell division and in the inhibition of apoptosis ${ }^{[20,21]}$. Of significance, survivin has aberrantly high expression in cancer cells such as lung cancer but little expression in most normal tissues, making survivin an attractive anticancer target ${ }^{[22,23]}$. Recent studies have shown that a designed oncolytic adenovirus driven by the survivin promoter exhibited a tumor-selective antitumor effect in vitro and in vivo ${ }^{[3,24,25]}$, suggesting that the survivin promoter is a good candidate for cancer therapy. To improve the OA tumor-specificity, a 24 bp region within the E1A conserved region 2 (CR2), which is responsible for binding the retinoblastoma $(\mathrm{Rb})$ protein, was deleted. This deletion restricts viral replication to dividing cells or Rb-inactive and arrested cells ${ }^{[3]}$. In this study, the dual-regulated Ad-sp-E1A ${ }_{(\Delta 24)}$ oncolytic virus contained the 24 bp deletion within E1A and was driven by the survivin promoter. Previous studies demonstrated that TSLC1, a candidate tumor suppressor in lung cancer, was depleted or not expressed in lung cancer cells ${ }^{[7,26]}$. Thus, it was inserted into the Ad·sp-E1A $\mathrm{A}_{(\Delta 24)} \mathrm{OA}$, yielding the Ad·sp-E1A $\mathrm{A}_{(\Delta 24)}$-TSLC1 construct. Our data indicated that Ad-sp-E1A ${ }_{(\Delta 24)}$-TSLC1 specifically induces dramatic cytotoxicity in lung cancer cells in vitro and effectively suppresses xenografted lung cancer in nude mice, suggesting that Ad·sp-E1A ${ }_{(\Delta 24)}$-TSLC1 may be a promising therapeutic agent for lung cancer.

\section{Materials and methods}

\section{Cell lines and culture conditions}

HEK293 (human embryonic kidney cell line containing the E1A region of Ad5) cell was obtained from Microbix Biosystem Inc (Toronto, Canada). All of the lung cancer cell lines (A549, NCI-H460, and H1299) and the normal lung cell line MRC-5 were obtained from American Type Culture Collection (ATCC, Rockville, MD, USA) or Shanghai Cell Collection (Shanghai, China). All cell lines were cultured in Dulbecco's modified Eagle's medium (DMEM) supplemented with 10\% heat-inactivated fetal bovine serum (FBS), $4 \mathrm{mmol} / \mathrm{L}$ glutamine, $50 \mathrm{U} / \mathrm{mL}$ penicillin and $50 \mathrm{mg} / \mathrm{mL}$ streptomycin. All cell lines were cultured at $37^{\circ} \mathrm{C}$ in $5 \% \mathrm{CO}_{2}$.

\section{Plasmids}

The pcDNA3-hygro-TSLC1 plasmid was graciously provided by Dr R STEENBERGEN at the Vrije Universiteit Medical Center (Amsterdam, Netherlands). The pXC2 adenovirus shuttle vector, $\mathrm{pMD}-\mathrm{T}$, and the $\mathrm{pBHGE3}$ adenoviral packaging vector were constructed in our laboratory. The $\mathrm{pXC2} \cdot \mathrm{sp}-\mathrm{E} 1 \mathrm{~A}_{(\Delta 24)} \mathrm{OA}$ plasmid was previously constructed in our laboratory ${ }^{[3]}$. The TSLC1 cDNA sequence was first cloned between the EcoR I and Xho I sites in the pMD-T vector to yield pMD-T-TSLC1. Then, $\mathrm{pXC2} \cdot \mathrm{sp}-\mathrm{E} 1 \mathrm{~A}_{(\Delta 24)}$-TSLC1 was constructed by inserting the entire TSLC1 expression cassette derived from pMD-T-

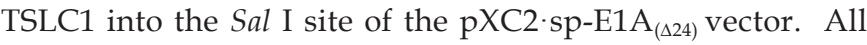
plasmid constructs were confirmed by restriction enzyme digestion, PCR and DNA sequencing.

\section{Quantitative RT-PCR}

Total RNA was isolated from prepared lung cancer cells or normal cells with TRIzol reagent (Invitrogen, USA) according to the manufacturer's instructions. For the analysis of Survivin and TSLC1 expression, cDNA was synthesized using Moloney murine leukemia virus reverse transcriptase (Invitrogen, USA) as described by the manufacturer. Quantitative real-time PCR was performed using a SYBR Green kit (TOYOBA, Japan). The glyceraldehyde-3-phosphate dehydrogenase (GAPDH) housekeeping gene was used for normalization. The following primers were used: TSLC1 forward primer, 5'-CGGCT- 
TCTGCTGTTGCTCTTCT-3'; TSCLC1 reverse primer, 5'-AAATAAATGGTCTGCCTGTTGG-3'; survivin forward primer, 5'-GACCACCGCATCTC-3'; and survivin reverse primer, 5'-AAGTCTGGCTCGTTC-3'. The RT-PCR was performed on an ABI Prism 7500 Sequence Detector (Applied Biosystems, USA). All of the reactions were conducted in triplicate. The $\Delta \Delta \mathrm{Ct}$ method was used for relative quantification of gene expression to determine survivin and TSLC1 mRNA expression.

Generation, identification, purification, and titration of adenovirus $\mathrm{Ad} \cdot \mathrm{sp}-\mathrm{E} 1 \mathrm{~A}_{(\Delta 24)}-\mathrm{TSLC1}$ and $\mathrm{Ad} \cdot \mathrm{sp}-\mathrm{E} 1 \mathrm{~A}_{(\Delta 24)}$ viral vectors were generated by homologous recombination of pXC2.sp-E1 $\mathrm{A}_{(\Delta 24)^{-}}$ TSLC1 and $\mathrm{pXC2} \cdot \mathrm{sp}-\mathrm{E} 1 \mathrm{~A}_{(\Delta 24)}$, respectively, with the PBHGE3 adenoviral packaging vector in HEK293 cells. Individual plaques were selected and used to infect HEK293 cells. After observing cytopathic effects, the cell culture medium was collected and viral genomic DNA was extracted. Then, wild-type adenovirus and foreign gene expression cassettes were identified by PCR methods using primer pairs complementary to the E1A region or an exogenous gene. Recombinant adenoviruses were amplified in HEK293 cells and purified by cesium chloride gradient ultracentrifugation. Viral titers were determined by TCID50 (median tissue culture infective dose) assays in HEK293 cells.

\section{Cell viability assay}

Cells were plated in 96-well plates and treated with different recombinant adenoviruses at the following MOIs: 0.5, 1, 2, 5, and 10 for $48 \mathrm{~h}$. Then, $20 \mu \mathrm{L}$ of MTT (Sigma, USA) solution $(5 \mathrm{mg} / \mathrm{mL})$ was added to each well. Cells were incubated at $37^{\circ} \mathrm{C}$ for $4 \mathrm{~h}$. The supernatant of each well was carefully removed, and an equal amount of DMSO $(150 \mu \mathrm{L})$ was added to each well and mixed thoroughly on a shaker for $10 \mathrm{~min}$. The absorbance of each well was read at $595 \mathrm{~nm}$ with a DNA microplate reader (GENios model, Tecan; Maennedorf, Switzerland).

\section{Cytopathic effect (CPE) assay}

NCI-H460, A549, and H1299 lung cancer cell lines and the normal fibroblast cell line MRC-5 were grown to subconfluence and infected with adenoviruses at various MOIs as described above. Six days after infection, a $2 \%$ crystal violet solution in $20 \%$ methanol was added to cells for $15 \mathrm{~min}$ and then washed with distilled water and photographed.

\section{Hoechst 33342 staining}

To detect chromatin condensation and nuclear fragmentation, which are characteristics of apoptosis, nuclei were stained with Hoechst 33342. A549, H1299, NCI-H460, and MRC-5 cells were infected with Ad·sp-E1A ${ }_{(\Delta 24)}$ and Ad·sp-E1A $\mathrm{A}_{(\Delta 24)}{ }^{-}$ TSLC1 viruses at an MOI of 10 for $72 \mathrm{~h}$. cells were fixed with $4 \%$ paraformaldehyde and then stained with the Hoechst 33342 staining kit (Beyotime, Nantong, China) for $20 \mathrm{~min}$ as described in the manufacturer's protocol. Cells were then washed twice with PBS and visualized under a fluorescence microscope. Uninfected cells served as a control.

\section{Western blot analysis}

Western blot analysis was performed using standard protocols to determine the expression of various proteins. Cells were trypsinized, harvested and resuspended in lysis buffer (62.5 $\mathrm{mmol} / \mathrm{L}$ Tris- $\mathrm{HCl}$ [pH 6.8], 2\% SDS, $10 \mathrm{mmol} / \mathrm{L}$ glycerol and $1.55 \%$ dithiothreitol). The total protein concentration was determined using the $\mathrm{BCA}^{\mathrm{TM}}$ Protein Assay kit (Pierce, Rockford, IL, USA) as described by the manufacturer. Then, protein samples were separated by 10\%-15\% SDS-polyacrylamide gel electrophoresis and transferred to nitrocellulose membranes (Millipore, MA, USA). Membranes were blocked in a 5\% BSA solution and incubated with primary antibodies. Proteins were detected using the appropriate secondary antibodies conjugated to fluorescent molecules and visualized with an Odyssey Infrared imaging system (LI-COR Biosciences Inc, Lincoln, NE, USA). Caspase- 3 and caspase- 8 antibodies were purchased from Cell Signaling Technology (Danvers, MA, USA). TSLC1, E1A, and PARP antibodies were obtained from Santa Cruz Biotechnology (Santa Cruz, CA, USA). The GAPDH antibody was purchased from Bioworld Technology (Bioworld, MN, USA).

\section{Animal experiments}

All of the animal experiments were approved by the Institutional Animal Care and Use Committee and were performed in accordance with the rules of our institution. Female BALB/c nude mice (4-5 weeks old) were purchased from the Shanghai Experimental Animal Center (Shanghai, China). A549 cells were subcutaneously injected into the lower right flank of female nude mice, and a tumor xenograft model was established. Each group was composed of at least eight animals, and tumor growth was monitored and measured every $3 \mathrm{~d}$ with a Vernier caliper. Tumor volume $(V)$ was calculated according to the formula: $V\left(\mathrm{~mm}^{3}\right)=1 / 2 \times$ length $(\mathrm{mm}) \times$ width $\left(\mathrm{mm}^{2}\right)$. When tumors reached an average volume of 100-130 $\mathrm{mm}^{3}$, mice were randomly allocated to six groups and given a daily dose of $5 \times 10^{8}$ plaque-forming units ( $\mathrm{pfu}$ ) of various adenoviruses in $100 \mu \mathrm{L}$ of PBS or PBS alone. Adenoviruses or PBS alone were administered intratumorally for $4 \mathrm{~d}$. Tumors were harvested $4 \mathrm{~d}$ after the last treatment for histopathologic and immunohistochemical (IHC) analyses.

\section{Immunohistochemistry}

For IHC analysis, tumors were harvested $4 \mathrm{~d}$ post-treatment and fixed in $4 \%$ paraformaldehyde, embedded in paraffin and cut into 4-mm sections. These sections were stained with goat monoclonal anti-adenoviral hexon antibody at a dilution of 1:200. Slides were then washed with PBS and incubated with the avidin-biotin-peroxidase complex (ABC) reagent (Vector Laboratories, Burlingame, CA, USA) and detected with diaminobenzidine tetrahydrochloride solution containing $0.006 \%$ hydrogen peroxide. Hematoxylin was used as a counterstain. Tissue sections stained without primary antibodies were used as negative controls. 
TdT-mediated dUTP-biotin nick end-labeling (TUNEL) assay

The TUNEL method was used to detect apoptotic cells. The in situ cell apoptosis detection kit (Roche, Palo Alto, USA) was used. The staining was performed according to the manufacturer's instructions. Tissue sections in the PBS-treated group were stained and served as negative controls. The TUNEL reaction preferentially labels DNA strand breaks generated during apoptosis and discriminates apoptosis from necrosis and primary DNA strand breaks induced by apoptotic agents.

\section{Transmission electron microscopy (TEM) analysis}

For electron microscopy analysis, tumor samples $\left(1 \mathrm{~mm}^{3}\right)$ were fixed in a PBS mixture containing $2.5 \%$ glutaraldehyde overnight and then incubated in $1 \%$ osmium tetroxide for $1 \mathrm{~h}$. Tissues were rinsed in $\mathrm{ddH}_{2} \mathrm{O}$, dehydrated through a graded series of ethanol and propylene oxide and finally embedded in Epon 812 resin (Shell Chemicals, Houston, TX, USA). After examination of semithin sections, areas were selected and subjected to ultrathin sectioning. Sections collected on 200 mesh copper grids were contrasted with lead citrate and uranyl acetate, examined and photographed with a JEOL 100CX transmission electron microscope (JEOL, Akishima, Japan).

\section{Statistical analysis}

The statistical significance of experimental results was calculated by the analysis of variance (ANOVA) and Student's $t$-test. All data are expressed as the meantstandard deviation (SD). Results were considered statistically significant at $P<0.05$.

\section{Results}

Characteristics of the oncolytic adenovirus Ad-sp-E1A ${ }_{(\triangle 24)}-$ TSLC1 To investigate the expression level of survivin and the TSLC1 gene, we first performed quantitative PCR. The results dem- onstrated that TSLC1 was significantly downregulated in several lung cancer cell lines (H1299, A549, and NCI-H460) compared to normal human fibroblast cells (MRC-5, Figure 1B). Conversely, survivin expression was cancer-specific and was detected in lung cancer cells (Figure 1A), which is consistent with previous reports ${ }^{[23,24]}$. Based on these results, we constructed the dual-regulated Ad·sp-E1A ${ }_{(\Delta 24)}$-TSLC1 viral vector in which the antitumor gene TSLC1 was inserted into $\mathrm{Ad} \cdot \mathrm{sp}-\mathrm{E} 1 \mathrm{~A}_{(\Delta 24)}$, which contains the survivin promoter and a 24 bp deletion within the E1A CR2 region (Figure 2A).
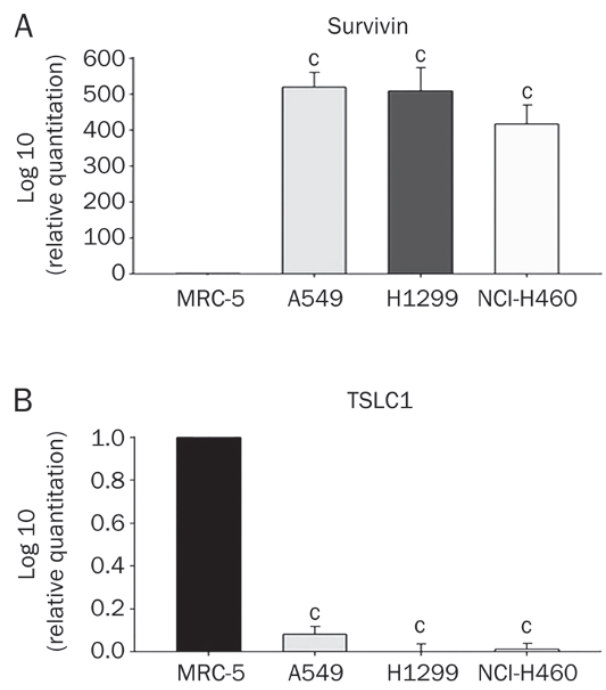

Figure 1. Relative expression level of survivin and TSLC1 in lung cancer cells. Survivin (A) and TSLC1 (B) mRNAs extracted from three lung cancer cell lines (H1299, A549, and $\mathrm{NCl}-\mathrm{H} 460)$ and human lung fibroblast cells line MRC- 5 were subjected to real-time quantitative PCR. Mean \pm SD. $n=3$. ${ }^{\mathrm{c}} P<0.01$.
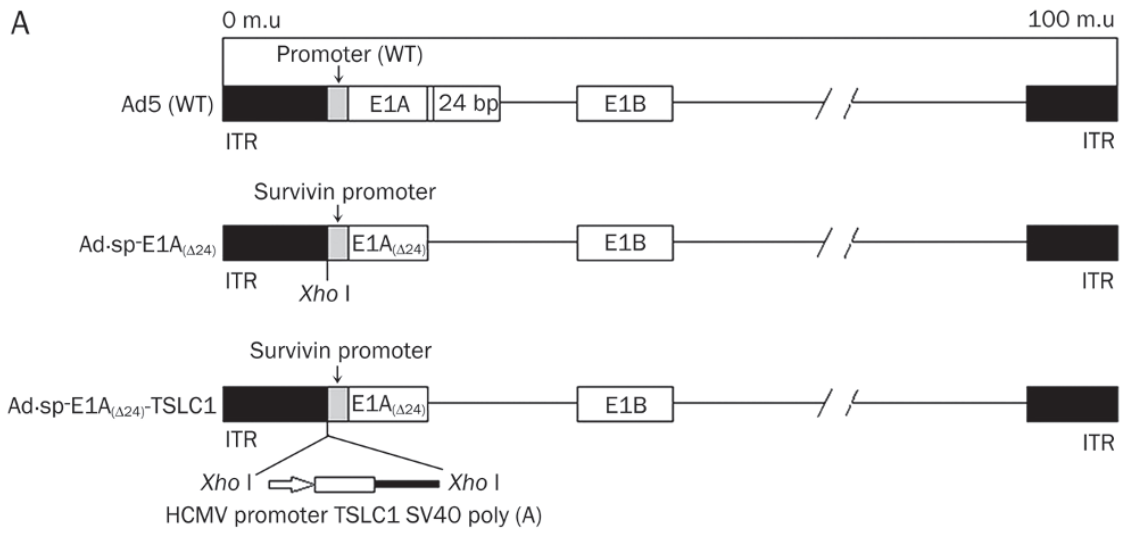

B
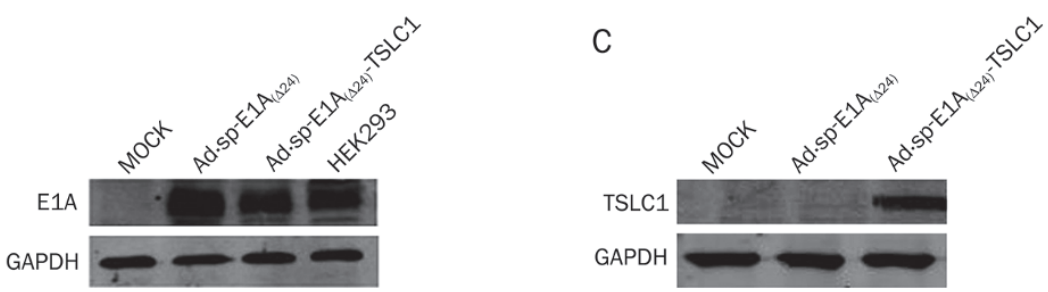

Figure 2. Characterization of oncolytic adenovirus Ad.sp-E1A $A_{(\Delta 24)}-T_{S L C 1}$. (A) Schematic diagram of recombinant oncolytic adenovirus structure. All viruses were constructed using the backbone of wild-type Ad5. ITR, inverted terminal repeat. Characterization of Ad-sp-E1A $A_{(\Delta 24)}$-TSLC1 was analyzed by Western blot. Lung cancer cell line A549 was infected with Ad.sp-E1A ${ }_{(\Delta 24)}-$ TSLC1 at a multiplicity of infection (MOI) of $10 \mathrm{pfu} / \mathrm{cell}$, and the E1A (B) and TSLC1 protein expression (C) was detected after $48 \mathrm{~h}$ by Western blotting analysis. 
We detected the tumor-specific expression of adenovirus E1A and the TSLC1 transgene. The A549 lung cancer cell line was infected with Ad·sp-E1A ${ }_{(\Delta 24)}$ and Ad·sp-E1A $A_{(\Delta 24)}$ TSLC1 at an MOI of 10 for $48 \mathrm{~h}$, and the expression of E1A and TSLC1 was then detected. These results indicated that both $\mathrm{Ad} \cdot \mathrm{sp}-\mathrm{E} 1 \mathrm{~A}_{(\Delta 24)}$ and Ad·sp-E1A $\mathrm{A}_{(\Delta 24)}-\mathrm{TSLC1}$ induced strong E1A expression (Figure $2 \mathrm{~B}$ ), implying that they replicated well in lung cancer cells. Additionally, the TSLC1 construct strongly induced TSLC1 expression compared to the mock treatment and $\mathrm{Ad} \cdot \mathrm{sp}-\mathrm{E} 1 \mathrm{~A}_{(\Delta 24)}$ control virus (Figure $2 \mathrm{C}$ ). These results demonstrate that the oncolytic virus can mediate TSLC1 expression in cancer cells.

Tumor cell-specific cytotoxicity mediated by Ad-sp-E1A $A_{(\triangle 24)}-T S L C 1$ in vitro

Next, we investigated the effect of Ad·sp-E1A ${ }_{(\Delta 24)}$ TSLC1 on cell viability. The human lung cancer cell lines A549, NCIH460, H1299, and the human normal fibroblast cell line MRC-5 were infected with Ad·sp-E1A ${ }_{(\Delta 24)}$ and Ad·sp-E1A $A_{(\Delta 24)}{ }^{-}$ TSLC1 at an MOI of 10, and cell proliferation was measured using the MTT assay. As shown in Figure 3, Ad·sp-E1A ${ }_{(\Delta 24)}$ TSLC1 induced cell death in approximately $48 \%$ to $65 \%$ of the infected cancer cells, and the tumor-killing effect of Ad·sp$\mathrm{E} 1 \mathrm{~A}_{(\Delta 24)}$-TSLC1 was more effective than Ad-sp-E1A ${ }_{(\Delta 24)}$ in a dose-dependent manner. In contrast, $90 \%$ of the MRC-5 cells were still viable after Ad·sp-E1A $A_{(\Delta 24)}$-TSLC1 infection. These results demonstrate the advantages of treating tumor cells with the dual-regulated oncolytic adenovirus.

Furthermore, the cytopathic effects induced by Ad.spE1A $_{(\Delta 24)}$-TSLC1 infections were visualized by crystal violet staining. Similar results were obtained by conducting the MTT assay on cancer cell lines treated with the various OAs for 4 d. As shown in Figure 4, significant cytopathic effects were
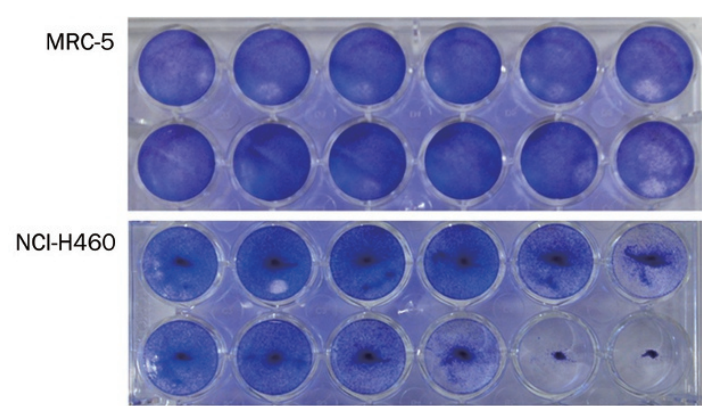

A549

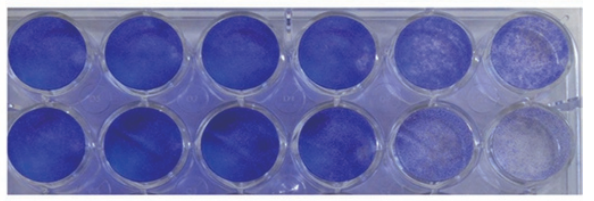

H1299
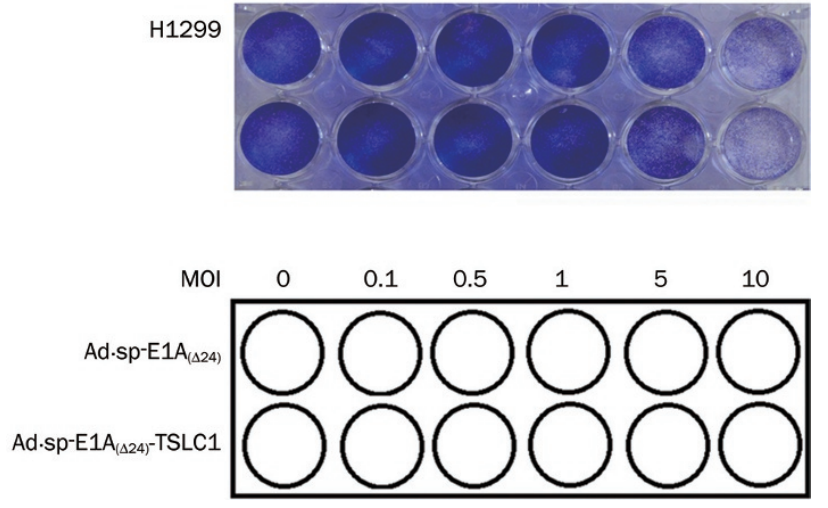

Figure 4. Tumor-specific cytopathic effect induced by Ad-sp-E1A $\mathrm{A}_{(\Delta 24)}-\mathrm{TSLC1}$. Three lung cancer cell lines (H1299, A549, and NCl-H460) and normal lung fibroblast cell lines MRC- 5 were seeded in 24-well plates as a density of $5 \times 10^{4}$ cells/well and infected with Ad.sp-E1A $\mathrm{A}_{(\Delta 24)}$ and Ad.sp-E1A $\mathrm{A}_{(\Delta 24)}$ TSLC1 at the indicated MOIs. Six days later, cells were stained with crystal violet.
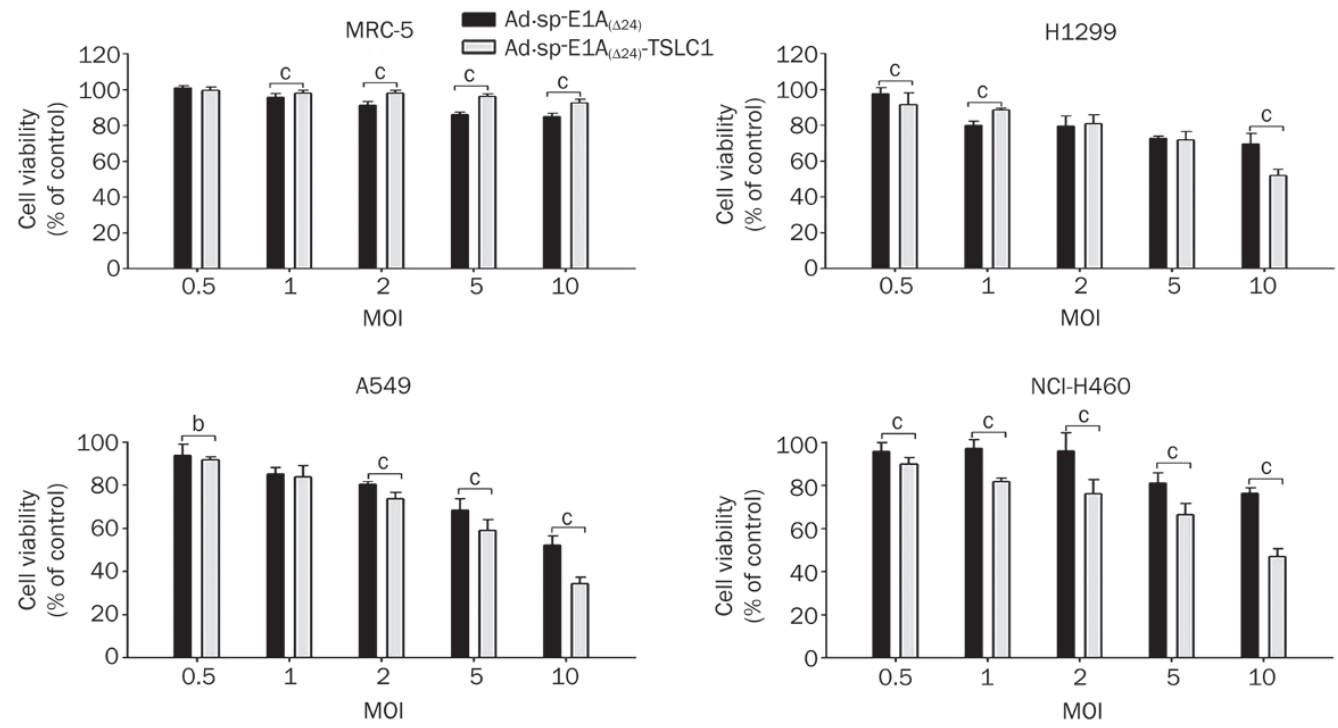

Figure 3. Suppression of tumor cell proliferation by Ad-sp-E1A ${ }_{(\Delta 24)}-\mathrm{TSLC1}$ in tumor cells in vitro. The lung cancer cell lines (H1299, A549, and NCl-H460) and normal lung fibroblast cell lines MRC-5 were infected with Ad.sp-E1A ${ }_{(\Delta 24)}$, and Ad.sp-E1A ${ }_{(\Delta 24)}-T_{S L C 1}$ at a MOI of 0.5, 1, 2, 5, and 10. Seventy-two hour later, cell viability rate was measured by MTT assay. Mean \pm SD. $n=4 .{ }^{b} P<0.05,{ }^{c} P<0.01$. 
observed in lung cancer cells infected with $\mathrm{Ad} \cdot \mathrm{sp}-\mathrm{E} 1 \mathrm{~A}_{(\Delta 24)^{-}}$ TSLC1, which mediated more cytopathic effects than Ad'sp$\mathrm{E} \mathrm{A}_{(\Delta 24)}$. Moreover, no obvious cytotoxicity was observed in normal cells under the same treatment conditions. Thus, the dual-regulated Ad-sp-E1 $A_{(\Delta 24)}-$ TSLC1 oncolytic virus could replicate selectively in lung cancer cells and induced tumorspecific cytotoxic effects.

\section{Ad.sp-E1A $A_{(\triangle 24)}-$ TSLC1 selectively induces cell apoptosis in vitro}

We also evaluated whether OA-mediated TSLC1 induces tumor-specific cell apoptosis in lung cancer cells. Treatment of cancer cells with Ad·sp-E1A ${ }_{(\Delta 24)}-$ TSLC1 led to increased apoptosis, which featured chromatin condensation, nuclear fragmentation and apoptotic bodies (Figure 5A). To assess whether the mechanism of apoptosis involved the caspase signaling pathway, Western blotting analysis was performed to detect the expression of caspase cascade proteins. Consistent with the above findings, increased activation of caspase-8, caspase-3 and PARP was detected in lung cancer cells treated with Ad·sp-E1A $A_{(\Delta 24)}$-TSLC1 compared to mock-treated or $\mathrm{Ad} \cdot \mathrm{sp}-\mathrm{E} 1 \mathrm{~A}_{(\Delta 24)}$-treated cells (Figure $5 \mathrm{~B}$ ). These results suggest that TSLC1 induces tumor cell apoptosis via activation of the caspase pathway.

\section{Antitumor activity of Ad.sp-E1A $\mathrm{A}_{(\triangle 24)}-\mathrm{TSLC1}$ in vivo}

The in vivo antitumor effects of Ad-sp-E1 $\mathrm{A}_{(\Delta 24)}$-TSLC1 were evaluated with a A549 xenograft model in nude mice. For all studies, mice with established tumors received percutaneous intratumoral injections of the viruses. Ad.sp-E1 $\mathrm{A}_{(\Delta 24)}$ and $\mathrm{Ad} \cdot \mathrm{sp}-\mathrm{E} 1 \mathrm{~A}_{(\Delta 24)}-\mathrm{TSLC} 1$ were injected as single doses of $5 \times 10^{8}$ pfu in a volume of $100 \mu \mathrm{L}$. Injections were given daily for $4 \mathrm{~d}$ to a group of mice $(n=8)$. PBS was used as a control. Tumor growth curves were plotted to compare the antitumor effects. As shown in Figure 6A, Ad·sp-E1A $\mathrm{( \Delta 24)}$ TSLC1 treatment significantly suppressed lung carcinoma development compared to PBS treatment $(P<0.05)$. Furthermore, Ad.sp-E1A $\mathrm{A}_{(\Delta 24)^{-}}$

A

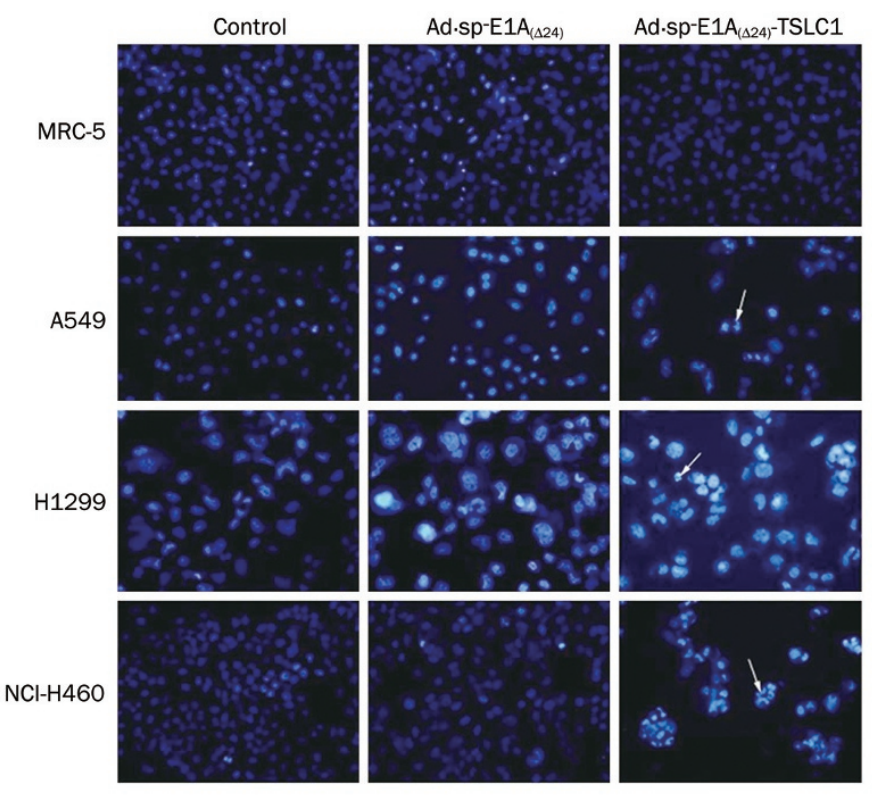

B

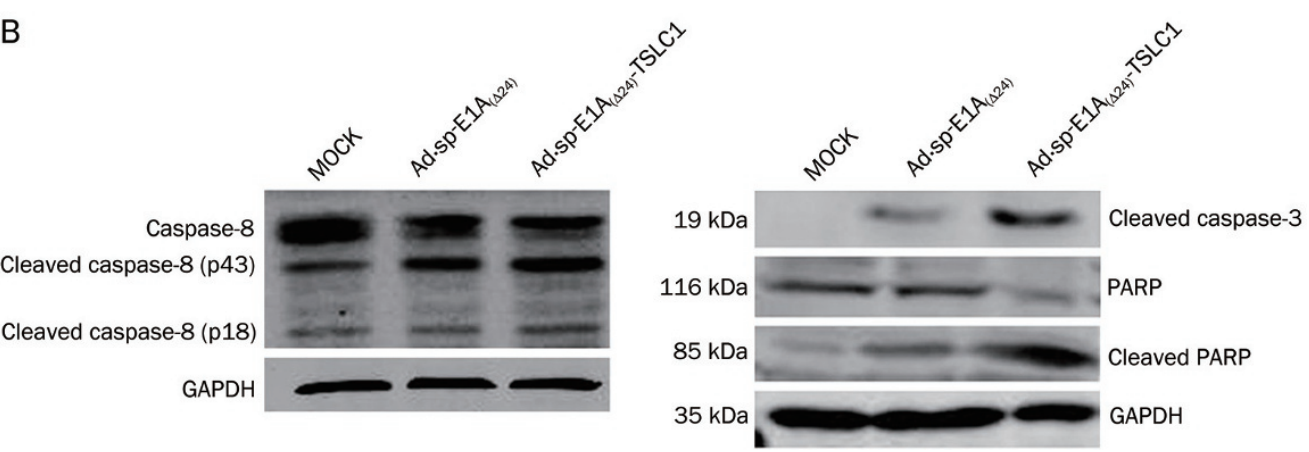

Figure 5. Detection of tumor cell apoptosis induced by Ad.sp-E1A $(\Delta 24)-T S L C 1$. (A) Apoptosis detection by Hoechst 33342 staining. Cells were plated

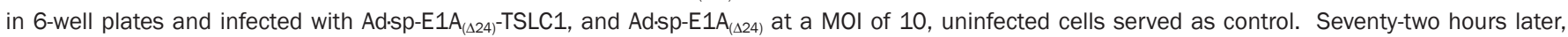
cells were treated with Hoechst33343 staining at $1 \mathrm{mg} / \mathrm{mL}$ for $30 \mathrm{~min}$, and then observed under the inverted fluorescence microscope. Original magnification, $\times 200$. (B) Activation of caspase signaling pathway by Ad.sp-E1A $A_{(\Delta 24)}-T_{S L C 1}$. The A549 cells were treated with the Ad·sp-E1A $\mathrm{A}_{(\Delta 24)}-\mathrm{TSLC1}$ at $10 \mathrm{MOI}$. Forty-eight hours later, cells were harvested and examined by Western blotting analysis. Activation of caspase-8, caspase-3, and the downstream apoptotic substrate protein poly (ADP-ribose) polymerase (PARP) was detected. GAPDH was used as the internal control. 

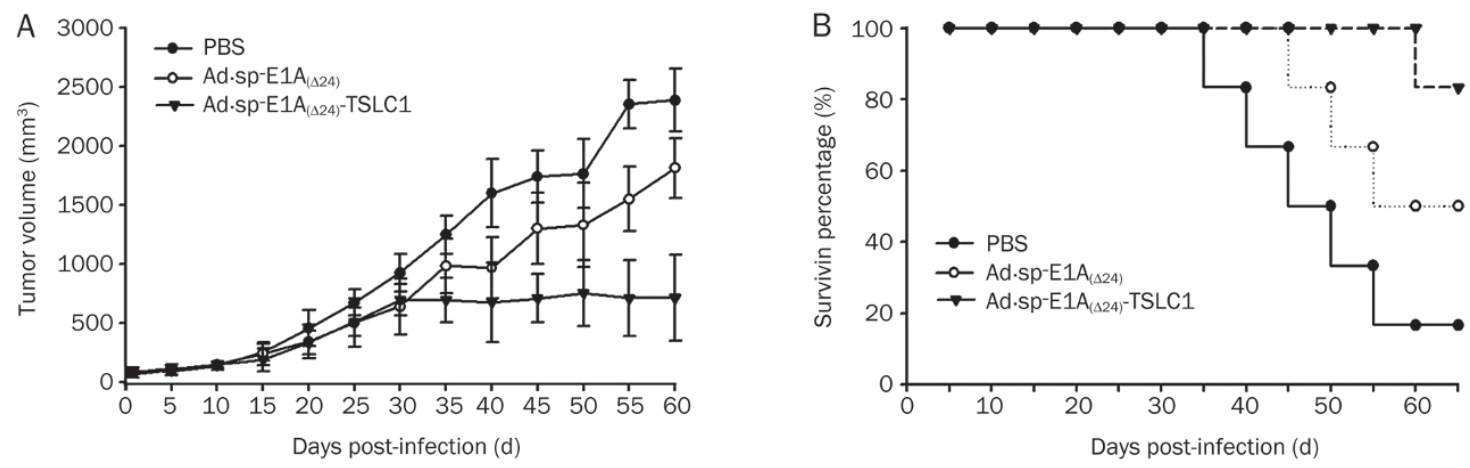

Figure 6. Antitumor effect of Ad-sp-E1A $\mathrm{A}_{(\triangle 24)}-\mathrm{TSLC1}$ in xenograft nude mice. Female BALB/c nude mice were subcutaneously inoculated with A549 cells $\left(5 \times 10^{6}\right)$. When tumors reached $100-130 \mathrm{~mm}^{3}$, the animals were treated with PBS, Ad·sp-E1A $A_{(\Delta 24)}$, or Ad.sp-E1A $A_{(\Delta 24)}-T_{S L C 1}$ through intratumoral injection. (A) Tumor volume of various treatment groups was measured. (B) Survival rate of mice was shown by the Kaplan-Meier survival curves. A pair-wise logrank test was used to analyze survival rates in the different groups. Mean \pm SD. $n=8$.

TSLC1 exhibited greater antitumor activity than Ad·sp-E1A $(\Delta 24)$

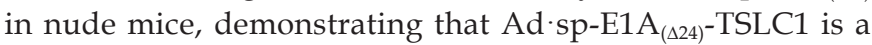
potent antitumor agent in vivo.

Survival of xenografted nude mice was monitored with a Kaplan-Meier curve (Figure 6B). Only one of the eight mice treated with Ad·sp-E1A ${ }_{(\Delta 24)}$-TSLC1 died within the first $65 \mathrm{~d}$. Conversely, PBS-treated mice gradually died after $35 \mathrm{~d}$, and the survival rate of these mice was less than $15 \%$. Furthermore, $50 \%$ of the Ad-sp-E1A ${ }_{(\Delta 24)}$-treated mice and $87.5 \%$ of the $\mathrm{Ad} \cdot \mathrm{sp}-\mathrm{E} 1 \mathrm{~A}_{(\Delta 24)}$-TSLC1-treated mice survived beyond the end of the experiment.

Pathological effects of Ad-sp-E1A $A_{(\Delta 24)}$-TSLC1 on tumor inhibition in nude mice

To detect cell death and the expression of TSLC1 and adenovirus hexon in tumor tissues, H\&E staining and IHC analysis utilizing anti-TSLC1 and anti-hexon antibodies were performed following various treatments. H\&E staining demonstrated that $\mathrm{Ad} \cdot \mathrm{sp}-\mathrm{E} 1 \mathrm{~A}_{(\Delta 24)}-\mathrm{TSLC} 1$ resulted in more severe cytopathic effects than Ad·sp-E1A $A_{(\Delta 24)}$ (Figure 7). IHC staining confirmed the strong expression of both TSLC1 and adenovirus hexon protein in the tumor tissues following treatment with Ad.spE1A $_{(\Delta 24)}$-TSLC1 (Figure 7), suggesting that the expression of TSLC1 increased as the oncolytic virus replicated in the tumor cells.

TUNEL assay results indicated that Ad·sp-E1A ${ }_{(\Delta 24)}$-TSLC1 treatment induced more extensive apoptosis in tumor tissue than Ad·sp-E1A ${ }_{(\Delta 24)}$ or PBS treatment (Figure 7). Morphological changes in tumor masses were also observed by TEM analysis (Figure 8A). Characteristics of apoptosis, including nuclear collapse, nuclear envelope disappearance, an increased nuclear-to-cytoplasmic ratio, nuclear deformation, the presence of heterochromatin and chromatin condensation were observed in tumors treated with Ad·sp-E1A ${ }_{(\Delta 24)}$ TSLC1. In addition, the presence and replication of $\mathrm{Ad} \cdot \mathrm{sp}-\mathrm{E} 1 \mathrm{~A}_{(\Delta 24)}$ and $\mathrm{Ad} \cdot \mathrm{sp}-\mathrm{E} 1 \mathrm{~A}_{(\Delta 24)}$-TSLC1 were observed in tumor tissues (Figure $8 \mathrm{~B})$. These results suggest that specific propagation of oncolytic viruses is involved in the inhibition of tumor growth.

\section{Discussion}

We developed the Cancer Targeting Gene-Viro-Therapy (CTGVT) strategy in $2001^{[27]}$, and this strategy has shown promising anticancer effects. Tumor-selective oncolytic viruses have been used as vectors to deliver the antitumor gene in CTGVT. The strategy improves cancer therapy through the dual action of the therapeutic gene and the oncolytic virus itself. The oncolytic poxvirus JX-594, which delivers granulocyte macrophage-colony stimulating factor (GM$\mathrm{CSF}$ ), exhibited outstanding antitumor results in a clinical trial via viral oncolysis and tumor-specific anti-cancer immunity ${ }^{[28]}$. JX-594 could selectively infect, replicate and express its transgene in cancer tissues but did not affect normal tissue; JX-594 was delivered into human metastatic solid tumors via intravenous infusion ${ }^{[29]}$.

The oncolytic adenovirus is the most customizable vector in clinical and preclinical studies for human cancer therapy due to several biological properties including ease of production, oncolysis ability and a large packaging capacity. Recently, additional modifications to the oncolytic virus were made to improve its targeting and application for cancer therapy. The early E1A protein is an essential factor for efficient adenoviral replication in host cells; thus, the modification of E1A is a preferred strategy. A 24 bp deletion within the E1A CR2 region allows the adenovirus to target the $\mathrm{Rb}$ pathway in cancer cells ${ }^{[30]}$. Moreover, the use of a tumor-specific promoter as a replacement for the E1A endogenous promoter improves the targeting of the oncolytic virus. The human telomerase reverse transcriptase (hTERT) promoter ${ }^{[31]}$, the a-fetoprotein $(\mathrm{AFP})$ promoter $^{[32,33]}$ and the differential display code 3 (DD3) promoter ${ }^{[34]}$ have been extensively utilized in targeted cancer therapy. Survivin is a cancer gene and could potentially be useful for cancer diagnosis and therapy ${ }^{[20,21,35]}$. A previous study demonstrated that the survivin promoter was superior for cancer targeting when compared to the hTERT promoter ${ }^{[24]}$. Thus, the Ad·sp-E1A $A_{(\Delta 24)}$ dual-regulated adenoviral vector was constructed with the survivin promoter and the 24-bpdeleted E1A gene to induce cancer-specific cytotoxic effects. 


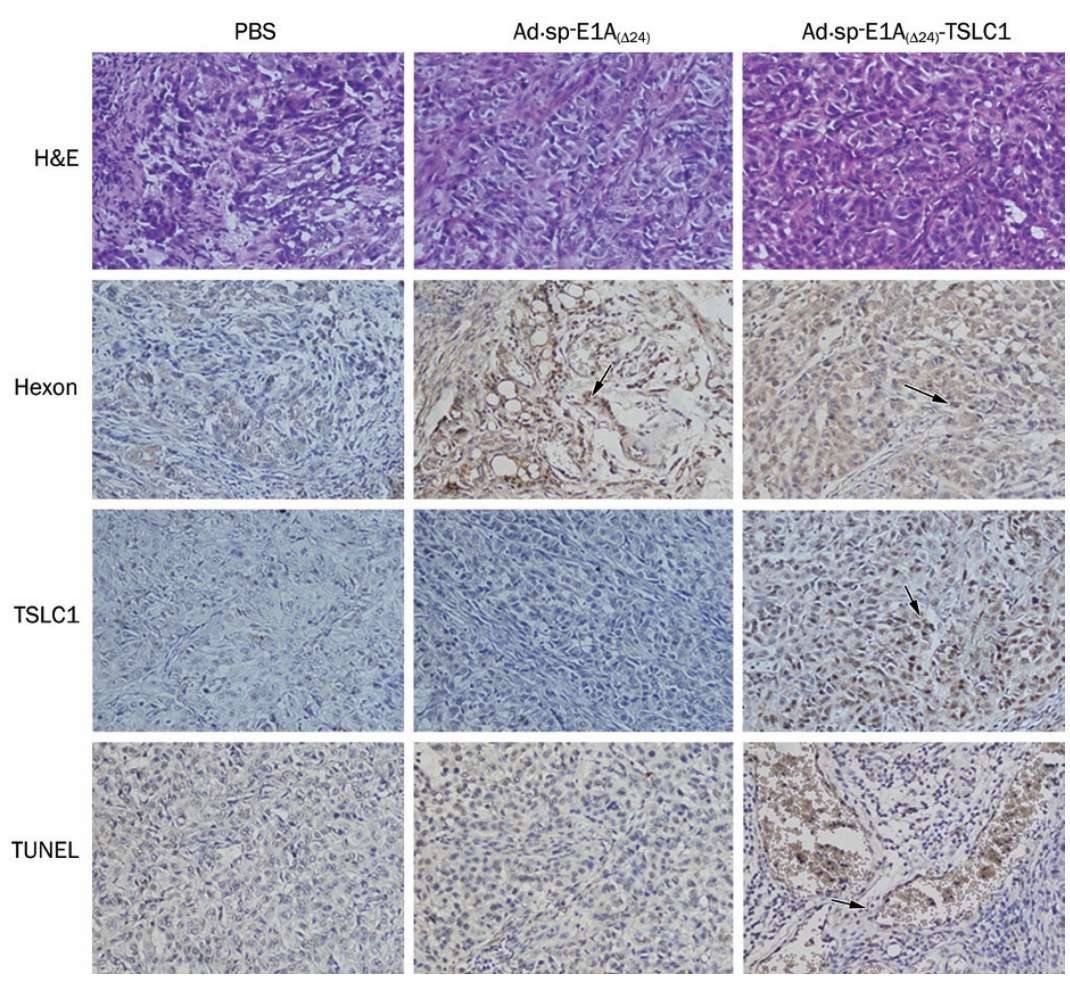

Figure 7. Ad.sp-E1A $(\triangle 24)-T S L C 1$ induced tumor cell death in in vivo lung cancer model by HE, IHC, and TUNEL assay. Mice xenograft A549 tumor tissues receiving various treatments were harvested and tumor sections were treated as described in Materials and methods. The most upper row is

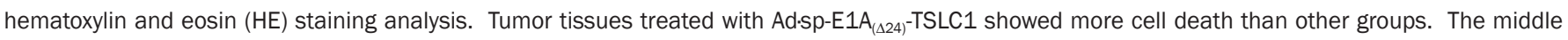
two rows showed adenovirus hexon and TSLC1 expression by IHC analysis in tumor tissues, respectively. The lowest row is TUNEL assay for detecting apoptotic cells treated with different treatment. Ad.sp-E1A $\mathrm{A}_{(\Delta 24)}-\mathrm{TSLC1}$ induced significant apoptosis of tumor cells. The brown color represents the apoptotic cells (as arrows shown). Original magnification: $\times 200$.
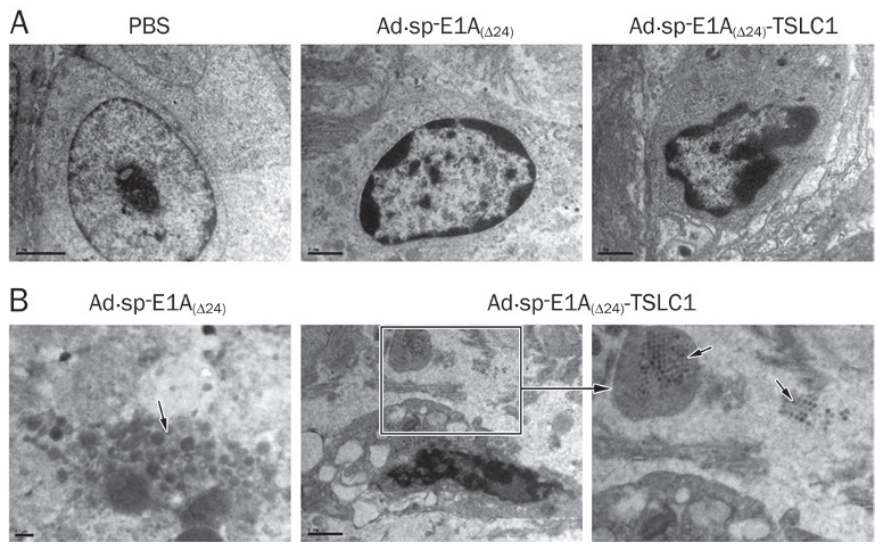

Figure 8. Morphological observation of tumor tissues by TEM analysis. (A) Morphological observation of cell apoptosis. The more obvious apoptotic phenomenon treated with Ad.sp-E1A $\mathrm{A}_{(\Delta 24)}$ TSLC1 was detected in tumor tissues than that of other treated groups, such as nuclear collapse, appearance of nucleus deformation, and the chromatin condensed in lumps et al. (B) Viral particles and replication (as arrows shown) in tumor tissues treated with Ad.sp-E1A ${ }_{(\triangle 24)}-\mathrm{TSLC} 1$.

In this study, Ad·sp-E1A $\mathrm{A}_{(\Delta 24)}-\mathrm{TSLC1}$ specifically replicated in lung cancer cells and induced cytotoxicity in those cells but not in normal cells (Figure 3, 4, and 6). Therefore, the survivin promoter-controlled E1A containing the 24 bp deletion may be useful for CTGVT in future lung cancer clinical trials.

Our results indicated that the expression of TSLC1 played a vital role in the cytotoxicity induced by Ad-sp-E1A $\mathrm{A}_{(\Delta 24)}-\mathrm{TSLC} 1$. TSLC1 is a cell adhesion molecule that potentially mediates the crosstalk between extracellular adhesion and intracellular signaling cascades through its interaction with additional tumor suppressors (eg, DAL4.1 and MPP1-3 $)^{[36]}$. Thus, TSLC1 mediates potential anti-cancer effects. Notably, our previous study reported that OA encoding TSLC1 exhibits significant antitumor effects on hepatocellular cancer cells ${ }^{[25]}$. The differences between these studies include the OA design and the cancer types investigated. The previous study utilized SD55-TSLC1, which contained TSLC1 inserted into the dualregulated OA; this vector also encoded E1A driven by the survivin promoter and included an E1B gene deletion. This construct was used to explore the inhibitory effect of TSLC1 on liver cancer growth. In this study, the oncolytic adenoviruses Ad·sp-E1A $\mathrm{A}_{(\Delta 24)}$ and Ad·sp-E1 $\mathrm{A}_{(\Delta 24)}$-TSLC1 suppressed lung tumors, but the latter exerted the strongest effect both in vitro and in vivo (Figure 3 and 6), indicating the powerful anticancer role of TSLC1. In addition, inactivating mutations in TSLC1 have been observed in human solid tumors. A mouse model containing a TSLC1 deletion was established, and these 
mice exhibited increased tumorigenesis and died significantly faster than wild-type controls ${ }^{[37]}$. These results confirm that TSLC1 is indeed an optimal tumor suppressor gene candidate for cancer gene therapy. Furthermore, we detected the activation of apoptosis via the caspase-dependent pathway, which was induced by oncolytic virus-mediated TSLC1 expression in lung cancer cells (Figure 5). Tumor growth of lung cancer xenografts was restricted to a small volume $30 \mathrm{~d}$ after Ad'sp$\mathrm{E} \mathrm{A}_{(\Delta 24)}-\mathrm{TSLC1}$ was injected into the nude mouse (Figure $6 \mathrm{~A}$ ), suggesting that Ad·sp-E1A $\mathrm{A}_{(\Delta 24)}-\mathrm{TSLC1}$ can be used as a potential treatment for lung cancer. In this study, high levels of TSLC1 expression were obtained by our dual-regulated oncolytic virus, which replicated selectively in lung cancer cells. The survivin promoter was employed to control E1A expression. The antitumor effects mediated by TSLC1 in our dualregulated oncolytic adenovirus were enhanced compared to the adenovirus-mediated TSLC1 that was previously reported for use in lung cancer ${ }^{[38]}$. This difference may be due to Ad.spE1A $\mathrm{A}_{(\Delta 24)}$-TSLC1-induced apoptosis and oncolysis by selective replication in lung cancer cells (Figure 8B). A recent study reported that the interaction of TSLC1 with its ligand CRTAM enhances the anti-tumor immune response to trichosanthin in a murine Lewis lung cancer model ${ }^{[39]}$. The role of TSLC1 in the activation of the host immune response requires further investigation. More recently, studies have reported that the TSLC1 gene is suppressed by microRNA-216a and microRNA$10 \mathrm{~b}$, facilitating early carcinogenesis, invasion and migration in hepatocellular carcinoma ${ }^{[40,41]}$. These other studies further validated the role of TSLC1 in cancer gene therapy.

In conclusion, we constructed a novel dual-regulated CTGVT oncolytic viral vector, Ad-sp-E1A $\left.A_{(\Delta 24)}\right)^{-T S L C 1, ~ c o n-~}$ taining a survivin promoter to control the expression of the E1A gene containing a $24 \mathrm{bp}$ deletion. Ad·sp-E1A $\mathrm{A}_{(\Delta 24)}-\mathrm{TSLC} 1$ displayed excellent antitumor effects in both lung cancer cells and in a nude mouse model. This report may provide a new strategy for the treatment of lung cancer.

\section{Abbreviations}

TSLC1, tumor suppressor of lung cancer 1; CTGVT, Cancer Targeting Gene-Viro-Therapy; SEER, Surveillance, Epidemiology and End Results; OA, oncolytic adenovirus; NSCLC, nonsmall-cell lung cancer; EMT, epithelial-mesenchymal transition; MOI, multiplicity of infection; MTT, 3-(4,5-Dimethylthiazol-2-yl)-2,5-diphenyltetrazolium bromide; CRTAM, Class I-restricted $\mathrm{T}$ cell-associated molecule.

\section{Acknowledgements}

This work was supported by the National Natural Science Foundation of China (№ 81272687), the Hi-Tech Research Development Program of China (863 Program, № 2012AA020806), the Open Funding Project of the State Key Laboratory of Bioreactor Engineering and Zhejiang Sci-Tech University Study Start-up grants (1016834-Y and 1016845-Y).

\section{Author contribution}

Yi-gang WANG designed the study; Wen LEI, Hong-bin LIU,
Bu-yun MA, and Yu-long XIA performed the research; Shibing WANG, Xiu-mei ZHOU, and Shui-di ZHENG contributed new reagents and analytic tools; Ke-ni GUO, Wen-song TAN, and Xin-yuan LIU analyzed data; Wen LEI and Yi-gang WANG wrote the paper.

\section{References}

1 Li Q, Tainsky MA. Epigenetic silencing of IRF7 and/or IRF5 in lung cancer cells leads to increased sensitivity to oncolytic viruses. PLoS One 2011; 6: e28683.

2 Liu XY. Targeting gene-virotherapy of cancer and its prosperity. Cell Res 2006; 16: 879-86.

3 Zhang KJ, Wang YG, Cao X, Zhong SY, Wei RC, Wu YM, et al. Potent antitumor effect of interleukin-24 gene in the survivin promoter and retinoblastoma double-regulated oncolytic adenovirus. Hum Gene Ther 2009; 20: 818-30.

4 Cao X, Yang M, Wei RC, Zeng Y, Gu JF, Huang WD, et al. Cancer targeting Gene-Viro-Therapy of liver carcinoma by dual-regulated oncolytic adenovirus armed with TRAIL gene. Gene Ther 2011; 18 : 765-77.

5 Zhao L, Gu J, Dong A, Zhang Y, Zhong L, He L, et al. Potent antitumor activity of oncolytic adenovirus expressing mda-7/IL-24 for colorectal cancer. Hum Gene Ther 2005; 16: 845-58.

6 Yu de B, Zhong SY, Yang M, Wang YG, Qian QJ, Zheng S, et al. Potent antitumor activity of double-regulated oncolytic adenovirus-mediated ST13 for colorectal cancer. Cancer Sci 2009; 100: 678-83.

7 Kuramochi M, Fukuhara H, Nobukuni T, Kanbe T, Maruyama T, Ghosh HP, et al. TSLC1 is a tumor-suppressor gene in human non-small-cell lung cancer. Nat Genet 2001; 27: 427-30.

8 Shingai T, Ikeda W, Kakunaga S, Morimoto K, Takekuni K, Itoh S, et al. Implications of nectin-like molecule-2/IGSF4/RA175/SgIGSF/ TSLC1/SynCAM1 in cell-cell adhesion and transmembrane protein localization in epithelial cells. J Biol Chem 2003; 278: 35421-7.

9 Honda T, Tamura G, Waki T, Jin Z, Sato K, Motoyama T, et al. Hypermethylation of the TSLC1 gene promoter in primary gastric cancers and gastric cancer cell lines. Jpn J Cancer Res 2002; 93: 857-60.

10 Tamura G. Promoter methylation status of tumor suppressor and tumor-related genes in neoplastic and non-neoplastic gastric epithelia. Histol Histopathol 2004; 19: 221-8.

11 Tamura G. Alterations of tumor suppressor and tumor-related genes in the development and progression of gastric cancer. World J Gastroenterol 2006; 12: 192-8.

12 Tsujiuchi T, Sugata E, Masaoka T, Onishi M, Fujii H, Shimizu K, et al. Expression and DNA methylation patterns of Tslc1 and Dal-1 genes in hepatocellular carcinomas induced by $\mathrm{N}$-nitrosodiethylamine in rats. Cancer Sci 2007; 98: 943-8.

13 Yang YX, Yang AH, Yang ZJ, Wang ZR, Xia XH. Involvement of tumor suppressor in lung cancer 1 gene expression in cervical carcinogenesis. Int J Gynecol Cancer 2006; 16: 1868-72.

14 Lung HL, Cheung AK, Xie D, Cheng Y, Kwong FM, Murakami Y, et al. TSLC1 is a tumor suppressor gene associated with metastasis in nasopharyngeal carcinoma. Cancer Res 2006; 66: 9385-92.

15 Heller G, Geradts J, Ziegler B, Newsham I, Filipits M, Markis-Ritzinger $\mathrm{EM}$, et al. Downregulation of TSLC1 and DAL-1 expression occurs frequently in breast cancer. Breast Cancer Res Treat 2007; 103: 283-91.

16 Fukuhara H, Kuramochi M, Fukami T, Kasahara K, Furuhata M, Nobukuni T, et al. Promoter methylation of TSLC1 and tumor suppression by its gene product in human prostate cancer. Jpn J Cancer Res 2002; 93: 605-9. 
17 Shimizu K, Onishi M, Sugata E, Fujii H, Honoki K, Tsujiuchi T. Aberrant DNA methylation of the $5^{\prime}$ upstream region of Tslc1 gene in hamster pancreatic tumors. Biochem Biophys Res Commun 2007; 353: 522-6.

18 Fukami T, Fukuhara H, Kuramochi M, Maruyama T, Isogai K, Sakamoto $\mathrm{M}$, et al. Promoter methylation of the TSLC1 gene in advanced lung tumors and various cancer cell lines. Int J Cancer 2003; 107: 53-9.

19 Murakami Y. Involvement of a cell adhesion molecule, TSLC1/IGSF4, in human oncogenesis. Cancer Sci 2005; 96: 543-52.

20 Ambrosini G, Adida C, Altieri DC. A novel anti-apoptosis gene, survivin, expressed in cancer and lymphoma. Nat Med 1997; 3: 917-21.

21 Adida C, Berrebi D, Peuchmaur M, Reyes-Mugica M, Altieri DC. Antiapoptosis gene, survivin, and prognosis of neuroblastoma. Lancet 1998; 351: 882-3.

22 Blanc-Brude OP, Mesri M, Wall NR, Plescia J, Dohi T, Altieri DC. Therapeutic targeting of the survivin pathway in cancer: initiation of mitochondrial apoptosis and suppression of tumor-associated angiogenesis. Clin Cancer Res 2003; 9: 2683-92.

23 Falleni M, Pellegrini C, Marchetti A, Oprandi B, Buttitta F, Barassi F, et al. Survivin gene expression in early-stage non-small cell lung cancer. J Pathol 2003; 200: 620-6.

24 Kamizono J, Nagano S, Murofushi Y, Komiya S, Fujiwara H, Matsuishi T, et al. Survivin-responsive conditionally replicating adenovirus exhibits cancer-specific and efficient viral replication. Cancer Res 2005; 65: 5284-91.

25 He G, Lei W, Wang S, Xiao R, Guo K, Xia Y, et al. Overexpression of tumor suppressor TSLC1 by a survivin-regulated oncolytic adenovirus significantly inhibits hepatocellular carcinoma growth. J Cancer Res Clin Oncol 2012; 138: 657-70.

26 Uchino K, Ito A, Wakayama T, Koma Y, Okada T, Ohbayashi C, et al. Clinical implication and prognostic significance of the tumor suppressor TSLC1 gene detected in adenocarcinoma of the lung. Cancer 2003; 98: 1002-7.

27 Liu XY. A novel anticancer strategy-Tumor Gene-viral therapy. Chin J Cancer Biother 2001; 8: 1. Chinese.

28 Hwang TH, Moon A, Burke J, Ribas A, Stephenson J, Breitbach CJ, et al. A mechanistic proof-of-concept clinical trial with JX-594, a targeted multi-mechanistic oncolytic poxvirus, in patients with metastatic melanoma. Mol Ther 2011; 19: 1913-22.

29 Breitbach CJ, Burke J, Jonker D, Stephenson J, Haas AR, Chow LQ, et al. Intravenous delivery of a multi-mechanistic cancer-targeted oncolytic poxvirus in humans. Nature 2011; 477: 99-102.
30 Fueyo J, Gomez-Manzano C, Alemany R, Lee PS, McDonnell TJ, Mitlianga $\mathrm{P}$, et al. A mutant oncolytic adenovirus targeting the $\mathrm{Rb}$ pathway produces anti-glioma effect in vivo. Oncogene 2000; 19: 2-12.

31 Su C, Na M, Chen J, Wang X, Liu Y, Wang W, et al. Gene-viral cancer therapy using dual-regulated oncolytic adenovirus with antiangiogenesis gene for increased efficacy. Mol Cancer Res 2008; 6: 568-75.

32 Hallenbeck PL, Chang YN, Hay C, Golightly D, Stewart D, Lin J, et al. A novel tumor-specific replication-restricted adenoviral vector for gene therapy of hepatocellular carcinoma. Hum Gene Ther 1999; 10: 1721-33.

33 Wei RC, Cao X, Gui JH, Zhou XM, Zhong D, Yan QL, et al. Augmenting the antitumor effect of TRAIL by SOCS3 with double-regulated replicating oncolytic adenovirus in hepatocellular carcinoma. Hum Gene Ther 2011; 22: 1109-19.

34 Ding M, Cao X, Xu HN, Fan JK, Huang HL, Yang DQ, et al. Prostate cancer-specific and potent antitumor effect of a DD3-controlled oncolytic virus harboring the PTEN gene. PLoS One 2012; 7: e35153.

35 Altieri DC. New wirings in the survivin networks. Oncogene 2008; 27 : 6276-84.

36 Sakurai-Yageta M, Masuda M, Tsuboi Y, Ito A, Murakami Y. Tumor suppressor CADM1 is involved in epithelial cell structure. Biochem Biophys Res Commun 2009; 390: 977-82.

37 van der Weyden L, Arends MJ, Rust AG, Poulogiannis G, Mclntyre RE, Adams DJ. Increased tumorigenesis associated with loss of the tumor suppressor gene Cadm1. Mol Cancer 2012; 11: 29.

38 Mao X, Seidlitz E, Truant R, Hitt M, Ghosh HP. Re-expression of TSLC1 in a non-small-cell lung cancer cell line induces apoptosis and inhibits tumor growth. Oncogene 2004; 23: 5632-42.

39 Cai Y, Xiong S, Zheng Y, Luo F, Jiang P, Chu Y. Trichosanthin enhances anti-tumor immune response in a murine Lewis lung cancer model by boosting the interaction between TSLC1 and CRTAM. Cell Mol Immunol 2011; 8: 359-67.

40 Li QJ, Zhou L, Yang F, Wang GX, Zheng H, Wang DS, et al. MicroRNA$10 \mathrm{~b}$ promotes migration and invasion through CADM1 in human hepatocellular carcinoma cells. Tumour Biol 2012; 33: 1455-65.

41 Chen PJ, Yeh SH, Liu WH, Lin CC, Huang HC, Chen CL, et al. The androgen pathway stimulates microRNA-216a transcription to suppress the TSLC1 tumor suppressor gene in early hepatocarcinogenesis. Hepatology 2012; 56: 632-43. 